\title{
Application of Real-Time PCR to Identify Residual Bio-Decontamination of Confined Environments after Hydrogen Peroxide Vapor Treatment: Preliminary Results
}

\author{
Michele Pazienza' ${ }^{1,3 *}$, Maria Serena Britti ${ }^{1,3}$, Mariachiara Carestia ${ }^{1 *}$, Orlando Cenciarelli ${ }^{1}$, Fabrizio D'Amico ${ }^{1,3}$, Andrea Malizia $^{1,3 *}$, Carlo $^{13}$
} Bellecci $^{1,3}$, Pasquale Gaudio ${ }^{1,3}$, Antonio Gucciardino ${ }^{3}$, Mariarosa Bellino ${ }^{1,3}$, Corrado Lancia ${ }^{1,3}$, Annalaura Tamburrini ${ }^{3}$ and Roberto Fiorito ${ }^{2,3}$

${ }^{1}$ Department of Industrial Engineering, University of Rome "Tor Vergata", Via del Politecnico 1 - 00133 Rome, Italy

2Department of Bio-Medical \& Prevention, School of Medicine and Surgery, University of Rome "Tor Vergata", Via Montpellier 1 - 00133 Rome, Italy

${ }^{3}$ International Master Courses in Protection against CBRNe events, Department of Industrial Engineering -School of Medicine and Surgery, University of Rome "Tor

Vergata", Italy

\begin{abstract}
This study was conducted to assess the effectiveness of Hydrogen Peroxide Vapor (HPV) to remove biological contamination in a confined environment and to evaluate real-time PCR assay as a technique for the evaluation of the decontamination efficiency. Decontamination after the dispersion of biological aerosol is a main issue from a civilian, public health and military perspective. Despite the effectiveness of aggressive substances, eco-friendly but still efficient methods for decontamination are a relevant demand and Hydrogen Peroxide Vapor (HPV) is among the most recent and promising technologies in this field. Another related issue is: when an environment can be considered fully decontaminated? The answer clearly depends on the objectives of the decontamination and this will affect the choice of the methodology.

Furthermore, classical microbiological and molecular biology techniques are commonly used to identify biological contamination and residual contamination, but many of them are time consuming and require advanced training for the operators who perform the analysis. This may represent a bottleneck, especially when a quick response to an emergency is needed (i.e. during an unconventional event like CBRNe ones). In this work, a combination of commercially available equipment for detection, identification and decontamination, was evaluated in partnership between the Italian Army, the Department of Industrial Engineering and the School of Medicine and Surgery of the University of Rome "Tor Vergata". The purpose of this work was to find a setup for equipment and methodologies for detection, identification and decontamination, to implement in case of biological events. Preliminary results show that, despite the death of the microorganisms, nucleic acids are not completely degraded by HPV treatment and, as a consequence, that real-time PCR may be the adequate, quick and easy method to verify the efficiency of bio decontamination when nucleic acid degradation represent the final objective.
\end{abstract}

Keywords: Hydrogen Peroxide Vapor; Real-Time PCR; Decontamination

\section{Introduction}

Contamination of confined environment with biological agents (i.e. toxins, vegetative bacteria, endospores, viruses, fungal and mold spores) is a main issue from both a civilian and military point of view.

From a "security" point of view, a biological event could be the result of the dissemination of biological agents due to a terrorist attack, routines operation in a healthcare setting [1-6] or to the outbreak of an infectious disease. In all those cases detection, identification and decontamination are among the main focuses. Key aspects, strictly related to this last aspect are: the bio-decontamination efficiency, the decontamination check system.

A biological event could affect a close environment, or in a wide open area, thus it becomes necessary to use an eco-friendly decontamination system: most available methods use substances, such as bleach or formaldehyde, which are each hazardous by themselves and produce further hazardous wastes. Advances in this field are represented by the use of hydrogen peroxide vapor (HPV) as surfaces decontaminant and sterilant [7] thanks to its anti-microbial effect due to the free radicals which acts on different biological molecules: lipids, proteins and nucleic acids [8].

HPV works well at low temperature and pressure, and is effective against a wide range of organisms including: bacterial endospores
[9], vegetative bacteria [10] and viruses [11]. It is safer than other disinfectant gases such as formaldehyde and ethylene oxide, and decomposes to water vapor and oxygen [12]. For this reason, HPV is currently used to decontaminate laboratory and medical equipment, pharmaceutical manufacturing facilities, and healthcare settings [1317], especially to reduce the risk of nosocomial infections [18-20].

Recent studies in this fields also proposes the use of HPV as

${ }^{*}$ Corresponding authors: Michele Pazienza, Department of Industrial Engineering University of Rome "Tor Vergata", Via del Politecnico 1 - 00133 Rome, Italy, E-mail: michele.pazienza@hotmail.it

Mariachiara Carestia, Department of Industrial Engineering, University of Rome "Tor Vergata", Via del Politecnico 1 - 00133 Rome, Italy, E-mail: mariachiara.carestia@uniroma2.it

Andrea Malizia, Department of Industrial Engineering, University of Rome "Tor Vergata", Via del Politecnico 1 - 00133 Rome, Italy, Tel: +39 0672597202 / +39 3666000132; E-mail: malizia@ing.uniroma2.it

Received November 15, 2013; Accepted December 23, 2013; Published December 30, 2013

Citation: Pazienza M, Britti MS, Carestia M, Cenciarelli O, D'Amico F, et al. (2013) Application of Real-Time PCR to Identify Residual Bio-Decontamination of Confined Environments after Hydrogen Peroxide Vapor Treatment: Preliminary Results. J Microb Biochem Technol 6: 024-028. doi:10.4172/1948-5948.1000116

Copyright: (C) 2013 Pazienza M, et al. This is an open-access article distributed under the terms of the Creative Commons Attribution License, which permits unrestricted use, distribution, and reproduction in any medium, provided the original author and source are credited 
Citation: Pazienza M, Britti MS, Carestia M, Cenciarelli O, D'Amico F, et al. (2013) Application of Real-Time PCR to Identify Residual BioDecontamination of Confined Environments after Hydrogen Peroxide Vapor Treatment: Preliminary Results. J Microb Biochem Technol 6 : 024-028. doi:10.4172/1948-5948.1000116

decontaminant for hazardous chemicals showing that HPV is able to produce changes in the structures of several pharmaceutical substances, although changes strongly depended on the chemical groups in the substance [21].

Several systems for HPV production are commercially available, and each shows differences and similarities. The VHP system by Steris (Basingstoke, UK) operates as a dry system, reducing the relative humidity inside the enclosure, so that condensation does not form on surfaces. The HPV system, by Bioquell (Andover, Hants, UK), injects the hydrogen peroxide vapor into the enclosure at such a level that micro-condensation forms on the internal surfaces. Therefore, the environment control is imperative for both systems [22]. Other comparative studies of hydrogen peroxide-based systems for airborne decontamination showed differences in efficacy, efficiency, and safety $[23,24]$, showing that technical aspects may strongly influence the decontamination results. All these aspects are to be considered when choosing the proper equipment for decontamination.

In case of intentional or unintentional release of biological agents, military forces and fire brigade are equipped and trained for detection identification and decontamination procedures of personnel and technical equipment. Since they have also molecular biology capability, an efficient decontamination of the equipment and the surrounding environment is paramount to avoid cross contamination issues [25].

The aim of this study was to simulate the diffusion of a biological aerosol in a confined environment, and to evaluate the efficiency of HPV as bio-decontaminant, with particular attention to the ability of HPV to degrade nucleic acids. With this aim, real-time PCR was proposed as a quick and easy technique to verify the residual biological contamination including that deriving from nucleic acids.

\section{Materials, Instruments and Methods}

The rationale of the trials is presented here. A solution containing Saccharomyces cerevisiae is aerosolized within a sealed chemical hood. Aerosol samples and samples of the Biological Indicator (BI), Geobacillus stearothermophilus, were collected before decontamination as positive control of the experiment. Two experiments were performed to evaluate differences in decontaminating with HPV a) immediately after the release of the aerosol or b) after 18 hours from the release.

At the end of the decontamination procedures, samples were collected and analyzed for cell viability; S. cerevisiae samples were further analyzed by real-time PCR to evaluate the presence of amplifiable target sequences.

\section{Strains}

A solution containing food grade $S$. cerevisiae lyophilized powder (Bertolini), at a concentration of $7 \mathrm{~g} / \mathrm{L}$, was aerosolized at a constant temperature of $25^{\circ} \mathrm{C}$ within a sealed chemical hood ( $1.5 \mathrm{~m}^{3}$ volume) by means of an automatic atomizer. This atomizer guarantees a flux of $0-70 \mathrm{~mL} / \mathrm{min}$ with a particle size in the range between 5 and 20 micron and the dispersion of the biological aerosol lasted for 3 minutes. It has to be clarified that S. cerevisiae is an "unofficial" biological warfare agent (BWA) substitute but it was chosen to guarantee safety of operators because of its non-pathogenicity, and also because of its structural characteristics which are in between prokaryotic and eukaryotic organisms (cell wall, nucleus, double strand linear chromosome). Besides, all the experiments were conducted using "Saccharomyces training kits" (Idaho Technology, Inc. now supplied as Bio Fire
Diagnostics, Inc), which are manufactured to safely perform laboratory routines in the same experimental conditions as using BWA samples.

G. stearothermophilus spores (purchased from BIOQUELL) have been added as positive control of decontamination. $G$. stearothermophilus spores are commonly used as biological indicator for decontamination due to their high resistance to heat and vapor $[26,27]$. G. stearothermophilus spores inoculated on steel carriers (10 $\mathrm{mm} \varnothing)$ to form a population of more than $1 \times 10^{6} \mathrm{CFU} /$ carrier. This BI's were placed on the most representative spots of the chemical hood i.e., the surface and the front glass of the hood for a total of 6 slides.

\section{Hydrogen peroxide vapor bio-decontamination}

Hydrogen peroxide vapor was produced and decomposed by the ClarusTM 'S' system (BIOQUELL). HPV was introduced within the hood by means of a double axis distribution system which guarantees an even distribution of HPV on the different surfaces; a schematic representation of the apparatus is shown in Figure 1. After 30 minutes of exposition, HPV was catalytically converted to water vapour and oxygen in the conversion phase which lasts 60 minutes, before continuation of operations.

\section{Sampling methods}

Samples from air and surfaces of the hood were collected before and after the decontamination procedure with HPV. Two different methods were used to sample the biological aerosol: a) active sampling of aerosol in air by means of a portable sampler (Fido 1 (FLIRTM), and b) passive sampling by positioning Petri dishes on the worktop of the hood.

The active sampler Fido 1 (FLIRTM) has a sampling flow rate of 200 liters per min and can collect particles whose size ranges between 0.5 and 10 microns.

Duration of sampling can be set by the operator according to the preset sampling duration: 5, 15, 30 and $60 \mathrm{~min}$. In this work, a sample

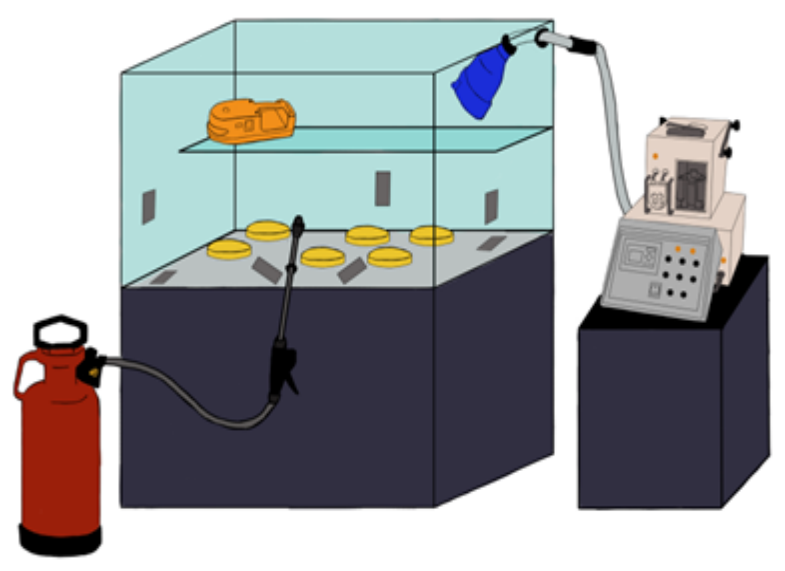

Figure 1: Representation of the experimental apparatus showing the chemical hood, the automatic atomizer (for the dispersion of $S$. cerevisiae) and the HPV production system. The system is sealed and no hydrogen peroxide vapor is allowed to escape. Petri dishes (in yellow) were placed on the worktop of the hood together with the automatic air sampler (orange), to collect samples of S. cerevisiae. Stainless steel indicators (grey rectangles) inoculated with $G$. stearothermophilus was placed on the worktop and on the internal walls of the hood. 
Citation: Pazienza M, Britti MS, Carestia M, Cenciarelli O, D'Amico F, et al. (2013) Application of Real-Time PCR to Identify Residual BioDecontamination of Confined Environments after Hydrogen Peroxide Vapor Treatment: Preliminary Results. J Microb Biochem Technol 6: 024-028. doi:10.4172/1948-5948.1000116

time of 5 minutes was chosen after considering the volume of air contained in a $1.5 \mathrm{~m}^{3}$ hood.

A 5 minutes sample duration, with a flow of 200 liters per min (for a total of 1000 liters of air) guarantees that the same air volume is not collected twice during the same sampling operation. Collected samples are then diluted in a water volume of $5 \mathrm{ml}$.

Passive sampling was performed by swabbing the 6 opened Petri dishes positioned on the worktop of the hood and plating swab on Agar Sabouraud [28].

Air and surface samples were collected at time intervals of an hour, and after about 24 hours from the release.

All samples were collected once, thus, the outcomes of this work are the result of single experiments.

\section{Culture media}

S. cerevisiae samples $(0.1 \mathrm{~mL}$ from $S$. cerevisiae samples collected immediately after aerosolization; $0.1 \mathrm{~mL}$ solution from air sampler and swab from the Petri dishes) were plated on Agar Sabauraud [28] and incubated at a temperature of $37^{\circ} \mathrm{C}$ for 48 hours.

G. stearotermophilus samples were inoculated in vials containing $10 \mathrm{~mL}$ of Tryptic Soy Broth (TSB) (BIOQUELL) and incubated at a temperature of $65^{\circ} \mathrm{C}$ for 7 days.

\section{Real-time PCR}

DNA extraction from the samples has been performed using the "IT 1-2-3DNA Sample purification kit" (BioFire Diagnostics, Inc).

Real-time PCR assay was conducted using the Ruggedized Advanced Pathogen Identification Device. The R.A.P.I.D.TM Bio Detection system (Idaho Technology Inc, now supplied as Bio Fire Diagnostics, Inc) is a ruggedized, portable real-time PCR designed to identify biological agents especially for mobile analytical labs and fields hospital.

The "S. cerevisiae Detection Kit for Hybridization Probe assay" (Idaho Technology, Inc., now supplied as Bio Fire Diagnostics, Inc.) was used for the identification of the agent; the kit consists of lyophilized reagents including primers which have specificity for $S$. cerevisiae and are validated according to the GMP (Good Manufacturing Practice) standards.

A fragment of the target DNA is amplified using specific primers. The amplicon is detected by fluorescence using a specific pair of hybridization probes. These probes consist of two different short oligonucleotides that hybridize to an internal sequence of the amplified fragment during the annealing phase of the reaction cycle. One probe is labeled at the $5^{\prime}$ end with LC Red 640 . To avoid extension on the 3 end, it is modified by phosphorylation. The second probe is labeled at the 3 ' end with fluorescein.

Only after hybridization to the template DNA do the two probes come in close proximity, resulting in fluorescence resonance energy transfer (FRET) between the two fluorophores.

The fluorescence emitted by the LC Red 640 dye is measured in channel 2 of the R.A.P.I.D. instrument.

The fluorescent signal from the unknown sample is compared to the signals from the positive and negative control samples.
Real-time PCR reactions were optimized at a temperature of $90^{\circ} \mathrm{C}$ for $5 \mathrm{sec}, 35$ amplification cycles at a temperature of $60^{\circ} \mathrm{C}$ for $15 \mathrm{sec}$. The melting curve analysis has been performed reaching a temperature of $90^{\circ} \mathrm{C}$ with a ramp of $0.2^{\circ} \mathrm{C} / \mathrm{sec}$.

For real-time PCR cycles settings, melting curve settings and sample preparation, protocols have been implemented according to the manufacturer's instructions.

\section{Results}

Real-time PCR assay, inoculation in liquid media of $G$. stearotermophilus spores and plating of $S$. cerevisiae were conducted for: a) decontamination with HPV immediately after the biological aerosol dispersion,

b) decontamination with HPV after 18 hours from the biological aerosol dispersion.

\section{Decontamination immediately after the release}

Real-time PCR assay on S. cerevisiae samples was conducted before and after the decontamination procedures. Results are shown in Table 1.

Decontamination efficiency has been tested on S. cerevisiae by plating samples collected both with the active and passive method (see section Materials, instruments and methods) on Agar Sabouraud and incubating the plates at a temperature of $37^{\circ} \mathrm{C}$ for 48 hours. After that the presence or the absence of microorganisms on the plate was evaluated. Results are shown in Table 2.

Decontamination efficiency was also tested on G. stearothermophilus spores used as biological indicator inside the hood. Steel carriers were collected after decontamination with HPV and inoculated in TSB. Table 3 shows results after an incubation period of 7 days at a temperature of $60^{\circ} \mathrm{C}$.

\section{Decontamination after 18 hours from the release}

Real-time PCR assay on S. cerevisiae samples was conducted before

\begin{tabular}{|l|c|c|}
\hline & $\begin{array}{c}\text { Sampling } \\
\text { method }\end{array}$ & $\begin{array}{c}\text { Real-time PCR } \\
\text { assay }\end{array}$ \\
\hline Before decontamination & SWAB & Positive \\
\hline \multirow{2}{*}{ After decontamination } & Fido 1 & Positive \\
\hline & SWAB & Positive \\
\hline
\end{tabular}

Table 1: Real-time PCR results before and after decontamination with HPV.

\begin{tabular}{|l|l|l|l|}
$\begin{array}{l}\text { Time } \\
(\mathrm{min})\end{array}$ & Swab & Air sampler & $\begin{array}{l}\text { S. cerevisiae culture after } \\
\text { Atomization (positive control) }\end{array}$ \\
\hline $\mathbf{0}$ & / & / & growth \\
\hline $\mathbf{5}$ & growth & growth & $/$ \\
\hline $\mathbf{1 3 5}$ & no growth & no growth & $/$ \\
\hline
\end{tabular}

Table 2: Swab from Petri dishes on the worktop and $0.1 \mathrm{~mL}$ of solution from air sampler plated on Agar Sabouraud at different timing during decontamination with SPV.

\begin{tabular}{lc|}
\hline Samples & Bacterial growth \\
\hline Positive control (Non treated) & Positive \\
Hood internal wall & Negative \\
Hood worktop & Negative \\
$\begin{array}{l}\text { Negative control (TBM not } \\
\text { inoculated) }\end{array}$ & Negative \\
\hline
\end{tabular}

inoculated)

Table 3: G. staerothermophilus growth on TSB after decontamination with HPV. 
Citation: Pazienza M, Britti MS, Carestia M, Cenciarelli O, D'Amico F, et al. (2013) Application of Real-Time PCR to Identify Residual BioDecontamination of Confined Environments after Hydrogen Peroxide Vapor Treatment: Preliminary Results. J Microb Biochem Technol 6: 024-028. doi:10.4172/1948-5948.1000116

and after the decontamination procedure which, in this case, started 18 hours after the dispersion of the yeast in the hood. Results are shown in Table 4

Decontamination efficiency on organisms vitality was tested on $S$. cerevisiae by plating samples collected both with the active and passive method on Agar Sabouraud and incubating the plates at a temperature of $37^{\circ} \mathrm{C}$ for 48 hours. Results are shown in Table 5 .

Steel carriers inoculated with G. stearothermophilus spores were collected after decontamination with HPV (18 hours after the release of biological aerosol) and inoculated in TSB. Results after an incubation period of 7 days at a temperature of $60^{\circ} \mathrm{C}$ are shown in Table 6 .

\section{Discussion}

According to the data collected from these preliminary trials, several aspects emerged. Decontamination with HPV showed similar results either when performed immediately after the release of $S$. cerevisiae and with a delay of 18 hours from the release, when almost all the aerosol is deposited on the worktop of the hood.

As a matter of fact, contamination evaluated by inoculation of samples (before and after the decontamination procedures) of $S$. cerevisiae, from active or passive sampling, shows the absence of microbial growth independently from weather decontamination is performed immediately after the release of the aerosol, or after 18 hours from the release. This result is also supported by the evaluation of bacterial growth after treatment with HPV of G. stearotermophilus in the same experimental conditions. No growth is observed from samples placed in the hood internal walls or work top when inoculating the steel carriers in TSB.

Controversial results were obtained from real-time PCR analysis. Real-time PCR on S. cerevisiae samples collected when decontamination is performed immediately after the dispersion of the

\begin{tabular}{|l|c|c|}
\hline \multirow{2}{*}{ Before decontamination } & Sampling Method & Real-time-PCR assay \\
\hline \multirow{2}{*}{ After decontamination } & SWAB & Negative \\
& Fido 1 & Positive \\
\hline & SWAB & Positive \\
\hline
\end{tabular}

Table 4: Real-time PCR results before and after decontamination with HPV.

\begin{tabular}{|l|l|l|l|}
$\begin{array}{l}\text { Time } \\
\text { (hours) }\end{array}$ & Swab & $\begin{array}{l}\text { Air } \\
\text { sampler }\end{array}$ & $\begin{array}{l}\text { S. cerevisiae culture after } \\
\text { atomization (positive control) }\end{array}$ \\
\hline $\begin{array}{l}\mathbf{0} \\
\text { (aerosol release) }\end{array}$ & $/$ & $/$ & growth \\
\hline $\mathbf{5} \mathbf{~ h}$ & growth & growth & $/$ \\
\hline $\mathbf{1 8} \mathbf{~ h}$ & growth & no growth & $/$ \\
\hline & Decontamination START \\
\hline $\mathbf{2 0} \mathbf{~}$ & Decontamination STOP \\
\hline $\mathbf{2 0 . 7 5} \mathbf{~ h}$ & no growth no growth \\
\hline
\end{tabular}

Table 5: Swab from Petri dishes on the worktop and $0.1 \mathrm{~mL}$ of solution from air sampler plated on Agar Sabouraud at different timing before and after decontamination with HPV.

\begin{tabular}{|l|c|}
\hline Samples & Bacterial growth \\
\hline Positive control (Non treated) & Positive \\
Hood internal wall & Negative \\
Hood worktop & Negative \\
Negative control (TBM not inoculated) & Negative \\
\hline
\end{tabular}

Table 6: G. staerothermophilus growth on TSB after decontamination with HPV (18 hours after the release of biological aerosol). yeast, show positive results (i.e. amplification of the sample) for the swab and negative results for air samples.

On the contrary, in case of decontamination after 18 hours from the dispersion of $S$. cerevisiae, amplification is present either in the sample collected from the petri dishes and from air.

This unexpected result may suggest that HPV is partially effective in degradation of $S$. cerevisiae nucleic acids when the yeast is still in the air fraction despite the fact that HPV is a surface sterilant; furthermore this would be true only if the positive result from decontamination after 18 hours can be attributed to a re-suspension of nucleic acids from the worktop of the hood. This last point may be explained considering the high sampling capacity of the air sampler versus the small volume of the air in the hood.

Nevertheless, real-time PCR seems to be a promising tool to detect residual contamination from biological agents (with the exception of toxins) since positive results were associated with negative growth on culture media for S. cerevisiae plated on Agar Sabouraud.

These results also suggest that, despite the ability of HPV to act as an antimicrobial agent, it cannot fully degrade nucleic acids. As a matter of fact, results show that the specific real-time PCR primers still recognize their target sequences of the microorganism.

Another relevant aspect that has to be better investigated is that the vitality of microorganisms may be affected by the atomization process and also by the sampling methods especially by the automatic ones.

The first aspect may not be a major issue since atomizers are used to simulate the atmospheric dispersion of biological agents in laboratory conditions, and our preliminary results show that $S$. cerevisiae samples collected before the decontamination procedures are fully able to grow on culture media but the sampling methods may significantly affect the results of the analysis.

Passive sampling efficiency strongly depends on the aerodynamic properties of the particles, which affect their deposition velocity, and the movement of air in the environment.

This method is not quantitative: it is not possible to correlate the number of microorganisms detected to the sampled air volume. The sensitivity is also low: this could explain the negative result of the realtime PCR on the sample collected by swabbing the petri dish before decontamination, although an error in the sampling preparation cannot be ruled out.

This observation is supported by the positive, result from realtime PCR performed before decontamination in the second trial (i.e. when decontamination is performed 18 hours after the aerosol release) which, can be considered a duplicate of the experiment.

Active sampling is more efficient and allows for correlation between the amounts of detected microorganisms to the sampled volume of air. On the other hand, it may mechanically stress the microorganisms, especially if they are not spores.

For this reason, a conventional microbiological analysis may not be able to identify viable but non-culturable [29] microorganisms.

In this last case, the contribution of real-time PCR techniques appears to be even more useful to identify a biological contamination and then, to verify the efficiency of the decontamination procedures.

\section{Conclusion}

An efficient decontamination in case of biological threats (both in 
Citation: Pazienza M, Britti MS, Carestia M, Cenciarelli O, D'Amico F, et al. (2013) Application of Real-Time PCR to Identify Residual BioDecontamination of Confined Environments after Hydrogen Peroxide Vapor Treatment: Preliminary Results. J Microb Biochem Technol 6 : 024-028. doi:10.4172/1948-5948.1000116

civilian and military contest) is a key issue for the safety of population and operators. In case of biological event, it is essential to have quick and easy tools to detect, identify and neutralize the biological threat. In this work the authors evaluated the efficiency of commercially available equipment to produce HPV, a more eco-friendly decontamination technique. This technique was used to decontaminate a confined environment after the dispersion of $S$. cerevisiae. Samples collected before and after the decontamination procedures showed that cellular growth on Agar Sabouraud is absent after a treatment with HPV for 30 minutes (independent on whether the decontamination procedure started immediately, or 18 hours after the aerosol dispersion). This efficacy is also achieved for G. stearothermophilus spores which are intrinsically more resistant to HPV.

Real-time PCR assay performed before and after decontamination showed positive results for the amplification of specific target sequences on S. cerevisiae genome. This suggests that treatment with HPV is not sufficient to degrade nucleic acids since they are still amplifiable by real-time PCR.

For this reason, in order to guarantee an efficient decontamination from nucleic acids and to prevent PCR carryover contamination, and contamination of samples and equipment, HPV decontamination should be supported by other decontamination techniques.

Real-time PCR assay revealed to be a quick, easy and effective method to identify contamination from biological agents, but also to verify the efficiency of the decontamination procedures when degradation of nucleic acid is required.

Further studies, including cost benefits analysis will be required to assess whether real-time PCR assay may be an implementation of the conventional techniques for the identification of residual biological contamination.

This approach is a clear example of a dual-use technique that can be translated from military to civilian world.

\section{Acknowledgments}

Special acknowledgments for the realization of this work go the International Master Courses in "Protection against CBRNe events" (www.mastercbrn.com).

\section{References}

1. Cenciarelli O, Malizia A, Marinelli M, Pietropaoli S, Gallo R, et al. (2013) Evaluation of biohazard management of the Italian national fire brigade. Defence S\&T Technical Bullettin 6: 33-41.

2. Gallo R, De Angelis P, Malizia A, Conetta F, Di Giovanni D (2013) Development of a georeferencing software for radiological diffusion in order to improve the safety and security of first responders. Defence S\&T Technical Bullettin 6: 21 32.

3. Malizia A , Lupelli I, D'Amico F, Sassolini A, Fiduccia A (2012) Comparison of software for rescue operation planning during an accident in a nuclear power plant. Defence S\&T Technical Bullettin 5: 36-45.

4. Malizia A, Quaranta R, Mugavero R, Carcano R, Franceschi G (2011) Proposa of the prototype RoSyD-CBRN, a robotic system for remote detection of CBRN agents. Defence S\&T Technical Bullettin 4: 64-76.

5. Malizia A, Quaranta R, Mugavero R (2010) CBRN events in the subway system of Rome: Technical-managerial solutions for risk reduction. Defence S\&T Technical Bullettin 2: 140-157.

6. Cenciarelli O, Rea S, Carestia M, D'Amico F, Malizia A, et al. (2013) Bioweapons and Bioterrorism: A Review of History and Biological Agents. Defence S\&T Technical Bullettin 6: 111-129.

7. Klapes NA, Vesley D (1990) Vapor-phase hydrogen peroxide as a surface decontaminant and sterilant. Appl Environ Microbiol 56: 503-506.
8. Hall L, Otter JA, Chewins J, Wengenack NL (2007) Use of Hydrogen Peroxide Vapor for Deactivation of Mycobacterium tuberculosis in a Biological Safety Cabinet and a Room. J ClinMicrobiol 45: 810-815.

9. Kokubo M, Inoue T, Akers J (1998) Resistance of common environmental spores of the genus Bacillus to vapor hydrogen peroxide. J Pharm SciTechnol 52: $228-231$

10. McDonnell G, Grignol G, Antloga K (2002) Vapour-phase hydrogen peroxide decontamination of food contact surfaces. Dairy Food Environ Sanit 22: 868873.

11. Heckert RA, Best M, Jordan LT, Dulac GC, Eddington DL, et al. (1997) Efficacy of vaporized hydrogen peroxide against exotic animal viruses. Appl Environ Microbiol 63:16-18.

12. Frencha GL, Otterb JA, Shannona KP, Adamsb NMT, Watling D, et al. (2004) Tackling contamination of the hospital environment by methicillin-resistant Staphylococcus aureus (MRSA): a comparison between conventional terminal cleaning and hydrogen peroxide vapour decontamination. J Hosp Infect 57 31-37

13. Rogers JV, Sabourin CL, Choi YW, Richter WR, Rudnicki DC, et al. (2005) Decontamination assessment of Bacillus anthracis, Bacillus subtilis, and Geobacillus stearothermophilus spores on indoor surfaces using a hydrogen peroxide gas generator. J Appl Microbio 99: 739-48.

14. Johnson JW, Arnold JF, Nail SL, Renzi E (1992) Vaporized hydrogen peroxide sterilization of freeze dryers. J Parenter Sci Technol 46: 215-225.

15. Jahnke M, Lauth G (1997) Biodecontamination of a large volume filling room with hydrogen peroxide. Pharm Engin 17: 96-108.

16. McDonnell G, Russell AD (1999) Antiseptics and disinfectants: activity, action and resistance. Clin Microbiol Rev 12: 147-179.

17. Krause J, McDonnell G, Riedesel H (2001) Bio-decontamination of anima rooms and heat-sensitive equipment with vaporized hydrogen peroxide. Contemp Top Lab Anim Sci 40: 18-21.

18. Kahnert A, Seiler P, Stein M, Aze B, McDonnell G, et al. (2005) Decontamination with vaporized hydrogen peroxide is effective against Mycobacterium tuberculosis. Lett Appl Microbiol 40: 448-52.

19. Passaretti CL, Otter JA, Reich NG, Myers J, Shepard J, et al. (2013) An evaluation of environmental decontamination with hydrogen peroxide vapor for reducing the risk of patient acquisition of multidrug-resistant organisms. Clin Infect Dis. Jan 56: 27-35.

20. Otter JA, Yezli S, Schouten MA, van Zanten ARH, Houmes-Zielman G, et al. (2010) Hydrogen peroxide vapor decontamination of an intensive care unit to remove environmental reservoirs of multidrug-resistant gram-negative rods during an outbreak. Am J Infect control 38: 754-756.

21. Švrček J, Syslová K , Stíbal D , Kuzma M , Kačer P (2012) Degradation of biologically active substances by vapor-phase hydrogen peroxide. Res Chem Int med. DOI 10.1007/s11164-012-0987-x.

22. Pottage T, Richardson C, Parks S, Walker JT, Bennett AM (2010) Evaluation of hydrogen peroxide gaseous disinfection systems to decontaminate viruses. J Hosp Infect 74: 55-61.

23. Fu TY, Gent P, Kumar V (2012) Efficacy, efficiency and safety aspects of hydrogen peroxide vapor and aerosolized hydrogen peroxide room disinfection systems. J Hosp Infect 80: 199-205.

24. Otter JA, Yezli S, Perl TM, Barbut F, French GL (2013) The role of 'no-touch' automated room disinfection systems in infection prevention and control. J Hosp Infect. 83: 1-13.

25. Mari G, Giraudi G, Bellino M, Pazienza M, Garibaldi C, et al. (2009) CBRN mobile laboratories in Italy. Proc. of SPIE Vol. 7304 73040W-1.

26. Feeherry FE,Munsey DT, Rowley DB (1987) Thermal inactivation and injury of Bacillus stearothermophilus spores. Appl Environ Microbiol53: 365.

27. Johnston MD, Lawson S, Otter JA (2005) Evaluation of hydrogen peroxide vapor as a method for the decontamination of surfaces contaminated with Clostridium botulinum spores. J Microbiol Methods 60: 403-411.

28. Sabouraud R (1896) Recherchedesmilieux de culture propres a la différenciation des espècestrichophytiques a grosse spore. Lestrichophytieshumaines. Paris: Masson et Cie, France 49-55.

29. Oliver JD (2005) The Viable but Nonculturable State in Bacteria. J Microbio 43: 93-100. 